\title{
Multi-jet production and jet correlations at CMS
}

\author{
Gábor I. Veres on behalf of the CMS Collaboration* ${ }^{\dagger}$ \\ CERN \\ E-mail: gabor.veresecern.ch
}

Hadronic jet production at the LHC is an excellent testing ground for QCD. Essential components of QCD, necessary for the description of the experimental data on hadronic jets, are hard parton radiation and multiple parton interactions. The importance of these components increases for final states including multiple jets. We will show results on observables sensitive to hard parton radiation, like the azimuthal (de)correlation between jets with small and large rapidity separation. Dijet events with a rapidity gap between them will also be presented, and their fraction measured as a function of jet transverse momentum and collision energy.

XXIV International Workshop on Deep-Inelastic Scattering and Related Subjects

11-15 April, 2016

DESY Hamburg, Germany

\footnotetext{
${ }^{*}$ Speaker.

${ }^{\dagger}$ The speaker thanks the support of CERN and the MTA-ELTE Lendület CMS Particle and Nuclear Physics Group.
} 


\section{Introduction}

There are several corners of the physics of strong interactions, where the fundamental theory describing most features of hadronic collisions, Quantum Chromodynamics (QCD), is challenging to apply. Various phenomenological models, approaches and approximations have been developed to describe these processes as generally as possible. Jet production in special configurations is an excellent testing ground for these models, and experimental data provides very important constraints on them. In order to successfully describe multi-jet production, one has to take into account hard parton radiation and multiple parton interactions. Correlation studies between jets and other particles are a useful tool to investigate the DGLAP and BFKL QCD evolution equations.

Recent measurements of observables sensitive to hard parton radiation will be presented, namely the azimuthal correlation between jets [1]. In addition, results on dijets with a rapidity gap, devoid of other charged particles, between them will be presented as well, demonstrating the role of the color singlet exchange process in the presence of a hard scale in the collision [2].

Both measurements have been performed by the CMS Collaboration [3], in proton-proton collisions at $\sqrt{s}=7 \mathrm{TeV}$. In both cases a special, low luminosity data collection period was necessary, as multiple proton-proton collisions occurring at the same time would constitute a large irreducible background or efficiency loss in these cases.

\section{Mueller-Navelet dijet decorrelations}

In the following, the most forward and backward jets (i.e. those with the largest $y$ on opposite sides of the interaction point) detected in a $\mathrm{p}+\mathrm{p}$ collision will be called Mueller-Navelet (MN) dijets. These jets have been reconstructed using the anti- $k_{T}$ algorithm, with a radius parameter $\mathrm{R}=0.5$. The calorimeter coverage allowed to reconstruct jets with axes in the interval $|y|<4.7$, leading to rapidity separations up to $\Delta y \approx 9.4$ [1]. The interest in the large separation is that while perturbative QCD calculations with collinear factorization using the Dokshitzer-Gribov-LipatovAltarelli-Parisi (DGLAP) parton evolution scheme are very successful in general, at low $p_{T}$ and large $\Delta y$ the Balitsky-Fadin-Kuraev-Lipatov (BFKL) evolution equations are expected to be more appropriate, predicting an increase of the dijet production cross section with increasing $\Delta y$.

While jets are produced back-to-back in azimuth $(\Delta \phi=\pi)$ on tree level, any decorrelation is due to higher-order processes, semi-hard parton interactions, and predicted to be sensitive to BFKL. A special trigger selecting forward-backward dijets with $|y|>3$ and $p_{T}>35 \mathrm{GeV}$ was employed to enhance the recorded data sample 100 -fold in this region. The average number of simultaneous $\mathrm{p}+\mathrm{p}$ collisions was 2.2 , and only those events with a single collision vertex were used. The data was unfolded for detector resolution effects. The migration between neighboring bins was kept below 20\%. The experimental observables were chosen to be the Fourier-harmonics (and their ratios) of the azimuthal angle difference between the two jets: $C_{n}=\langle\cos (n(\pi-\Delta \phi))\rangle$. For back-to-back jets one obtains $C_{n} \approx 1$, while decorrelations between jets will suppress this value.

The results have been compared to various Monte Carlo models. Some of these include DGLAP and leading-log (LL) soft and collinear radiation, tuned to LHC data (PYTHIA6 Z2, PYTHIA8 4C, HERWIG++), or DGLAP with tree-level matrix elements and LL parton showers (SHERPA), or include next-to-leading order (NLO) terms (POWHEG interfaced with PYTHIA); 

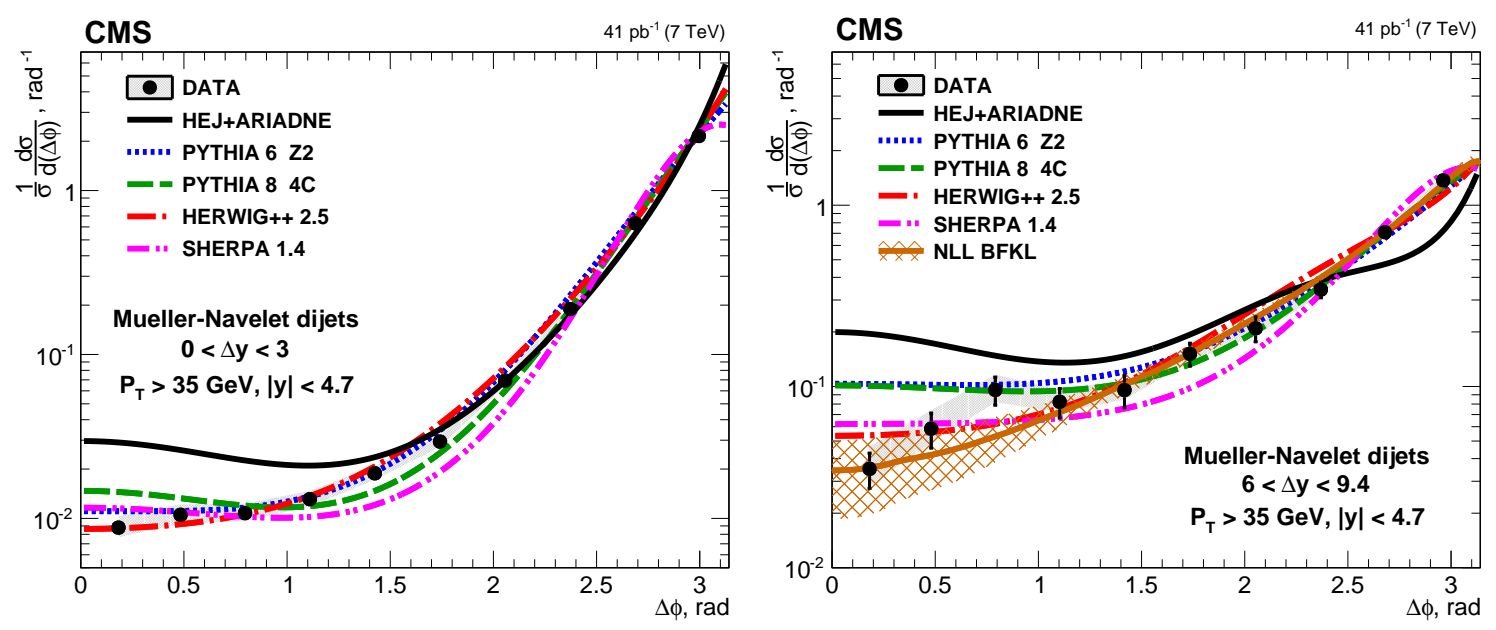

Figure 1: Left: Distribution of the azimuthal-angle difference, $\Delta \phi$, between $\mathrm{MN}$ jets in the rapidity interval $\Delta y<3.0$ [1]. The data (points) are plotted with experimental statistical (systematic) uncertainties indicated by the error bars (the shaded band), and compared to predictions from the leading-log DGLAP-based MC generators PYTHIA6, PYTHIA8, HERWIG++ and SHERPA, and to the leading-log BFKL-motivated MC generator HEJ with hadronization performed with ARIADNE (solid line). Right: the same for the rapidity interval $6<\Delta y<9.4$, including an analytical NLL BFKL calculation.

while there are also analytical next-to-leading-log (NLL) BFKL predictions [4] and a model using LL BFKL matrix elements (HEJ).

The left panel of Fig. 1 shows the $\Delta \phi$ distribution of dijets in the range $\Delta y<3$, exhibiting a sharp correlation, and that DGLAP models like PYTHIA6 and HERWIG++ agree well with the data; PYTHIA8 and SHERPA significantly deviate from them and HEJ clearly overestimates decorrelations at low $\Delta \phi$.

The back-to-back correlations become quite diluted when the two jets are separated by a large rapidity interval, as the right panel of Fig. 1 illustrates. The PYTHIA models visibly deviate from the data at small $\Delta \phi$ values, as opposed to small rapidity separations.

We shall turn to average cosine measurements now as a function of rapidity separation, thereby characterizing azimuthal dijet decorrelations with a single value in each $\Delta y$ interval. Average cosines also have the advantage that they can be expressed using conformal symmetries in the BFKL formalism. The left panel of Fig. 2 shows the $\langle\cos (\pi-\Delta \phi)\rangle$ averages as a function of the $\Delta y$ separation between the two jets, compared to three model calculations. Among these, SHERPA with parton matrix elements matched to a LL DGLAP parton shower does not predict sufficient amount of decorrelation, while the LL BFKL inspired generator HEJ with hadronization by ARIADNE overestimates it. The analytical NLL BFKL calculation at the parton level, presented by the solid red line, exhibits a fair agreement with the data. Further studies show that the $C_{2}$ coefficient is also underestimated by HEJ and overestimated by SHERPA at large rapidity separations, while the analytical calculation is successful.

One can also evaluate various ratios of the $C_{n}$ harmonics. These ratios suppress DGLAP contributions and uncertainties of factorization and renormalization scales. The right panel of Fig. 2 shows the measured ratio $C_{2} / C_{1}$ as a function of $\Delta y$, compared to LL DGLAP parton shower gener- 

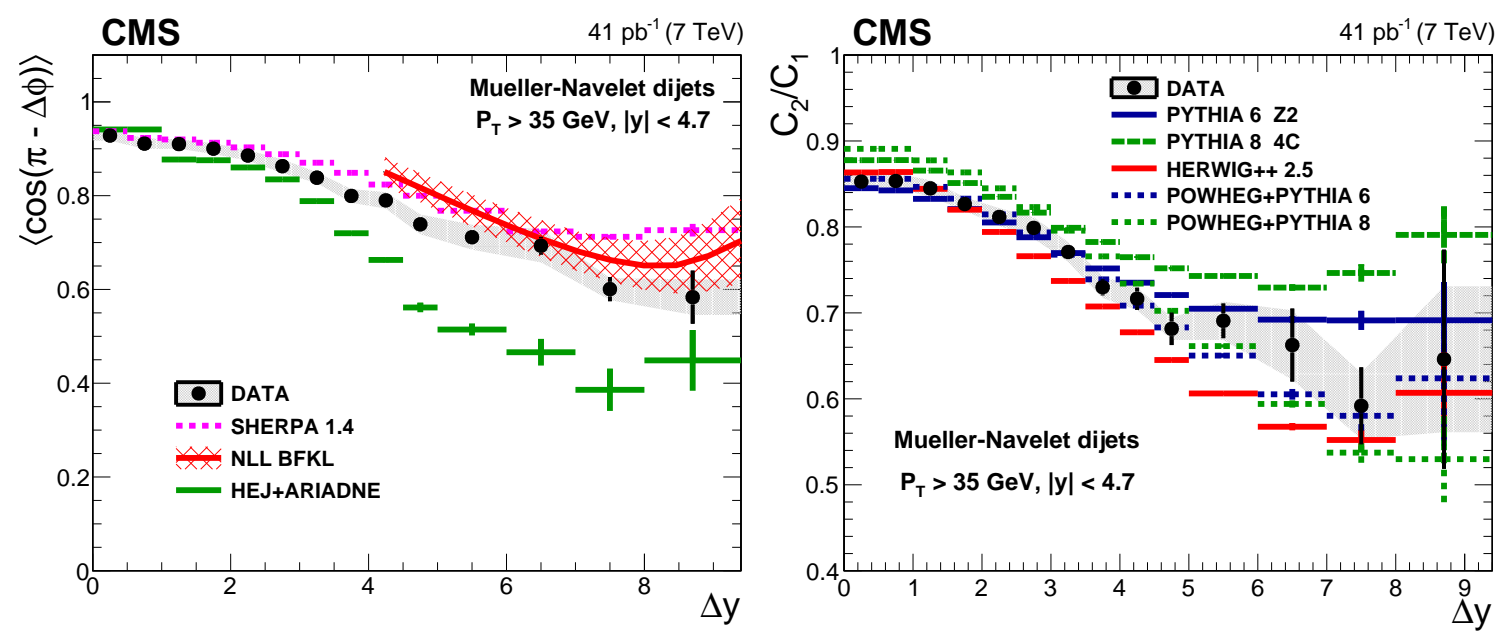

Figure 2: Left: Average $\langle\cos (\pi-\Delta \phi)\rangle$ as a function of $\Delta y$ [1] compared to the MC generator SHERPA with parton matrix elements matched to a LL DGLAP parton shower, to the LL BFKL inspired generator HEJ with hadronization by ARIADNE, and to analytical NLL BFKL calculations at the parton level $(4.0<$ $\Delta y<9.4)$. Right: The measured ratio $C_{2} / C_{1}$ as a function of rapidity difference $\Delta y$ compared to LL DGLAP parton shower generators and to the NLO generator POWHEG interfaced with PYTHIA6 and PYTHIA8.

ators and to the NLO generator POWHEG interfaced with PYTHIA6 and PYTHIA8. The PYTHIA event generators overestimate this ratio, while HERWIG underestimates it. Further studies indicate that the NLL BFKL calculation agrees with the data [1].

Multi-parton interactions (MPI) are expected to represent another important source of dijet decorrelations. Thus it is interesting to note that neither PYTHIA8 nor HERWIG++ predicts any significant difference of average cosine harmonics (or their ratios) between MPI turned on and off in these event generators.

In summary, the experimental data are well described by DGLAP MC models with LL parton shower and color coherence effects (HERWIG++). POWHEG with NLO calculations interfaced with PYTHIA does not constitute a significant improvement over PYTHIA alone concerning these observables. The HEJ model generally overestimates decorrelations, while analytical NLL BFKL calculations agree with data well.

\section{Dijet events with a rapidity gap}

Dijets are normally produced via a gluon or quark exchange, and the resulting color field between partons leads to emission of additional particles between the jets, usually described by the ( $k_{T}$ ordered) DGLAP formalism. In some cases there is no particle production activity between jets, and the BFKL formalism (using ordering in $x$ ) is more appropriate. The absence of particles in a large rapidity interval in hadron-hadron collisions is often associated with diffractive processes and color singlet exchange (CSE).

In the following experimental investigation of CSE, a search for events with the characteristic signature of two leading jets with no other charged particles in between them will be presented [2]. Jets were required to have more than $40 \mathrm{GeV}$ transverse momentum and lie in the $1.5<|y|<4.5$ 

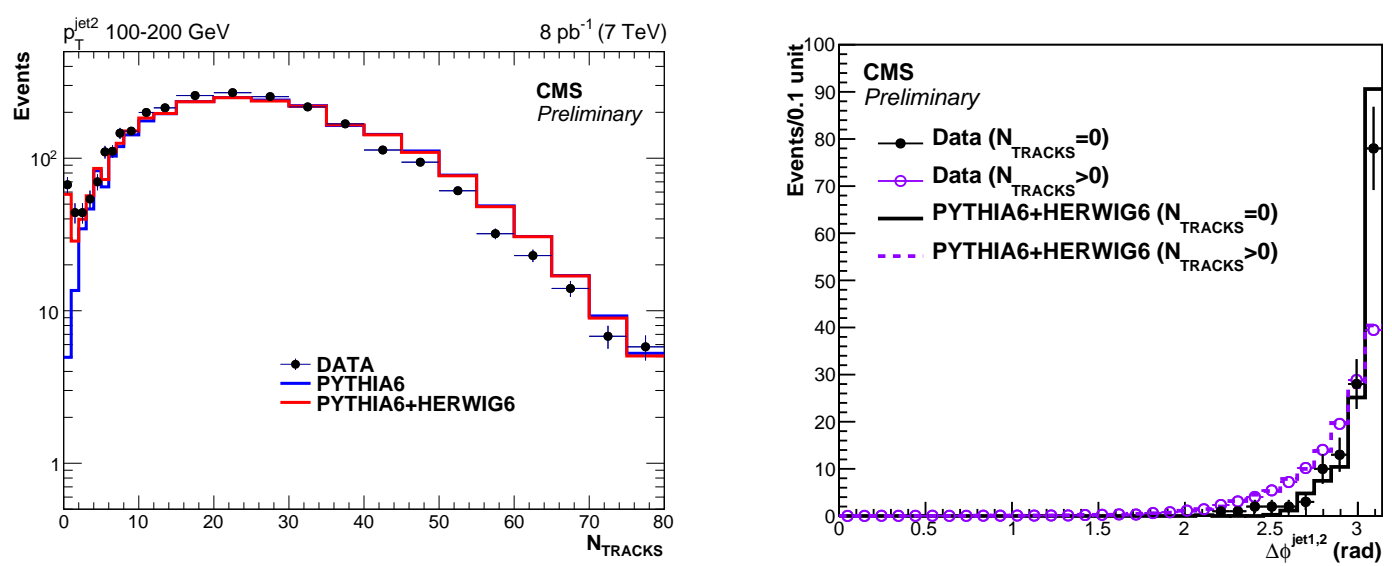

Figure 3: Left: Charged multiplicity distributions in the $|\eta|<1$ interval for $100<p_{T}^{\text {jet2 }}<200 \mathrm{GeV}$ [2], compared to predictions of PYTHIA6 and HERWIG6. The normalization of the MC distributions is obtained from a fit to the data. In the HERWIG model, only the CSE process is turned on. Right: Distribution of the azimuthal angle $\Delta \phi^{\text {jet1,2 }}$ between the two leading jets for events after all selections, with no tracks $\left(\mathrm{N}_{\text {TRACKS }}=0\right.$, filled circles) or at least one track ( $\mathrm{N}_{\text {TRACKS }}>0$, open circles) reconstructed in the $|\eta|<1$ region, compared with $\mathrm{MC}$ predictions. The distributions are normalized to the number of events in the data sample corresponding to $\mathrm{N}_{\mathrm{TRACKS}}=0$.

rapidity interval. Such jet-gap-jet events have earlier been observed by the D0 and CDF experiments at the Tevatron, and also at HERA. In the study presented here, the gap is defined by the pseudorapidity interval $|\eta|<1$ and charged particles are required to have $p_{T}>0.2 \mathrm{GeV}$.

The left panel of Fig. 3 shows the charged particle multiplicity distribution in the gap between the two leading jets. This distribution is well described by DGLAP models (PYTHIA6 in this case) except at zero multiplicity, i.e. in case of jet-gap-jet events. The excess of events visible at $N_{\text {TRACKS }}=0$ however agrees well with the CSE cross section predicted by the HERWIG6 model.

The right panel of Fig. 3 illustrates that the distribution of the azimuthal angle difference between jets depends on the multiplicity (zero or non-zero) in the gap between them, as expected. Jets with the CSE signature are more back-to-back (and also more balanced). These distributions are predicted quite precisely by Monte Carlo models, including color singlet exchange processes. In the zero-multiplicity event category, the BFKL-based Monte Carlo generator describes the $p_{T}$ distributions of the leading (highest $p_{T}$ ) and subleading jet as well [2].

The background of non-CSE events can be subtracted in various ways. One simple method is a negative binomial distribution fit to the charged particle multiplicity distribution in the gap, excluding the CSE peak at (around) zero and extrapolated to the low multiplicity region. The result of this subtraction can be seen on the left panel of Fig. 4. The excess of events in the peak is consistent with the prediction of the HERWIG6 model using the color singlet exchange process.

The number of events in the CSE peak can then be evaluated by integration over the first few $N_{\text {TRACKS }}$ bins. A fraction of these CSE dijet events among all dijet events can be calculated, and it is on the order of $0.5-1 \%$ in proton-proton collisions at $\sqrt{s}=7 \mathrm{TeV}$. The fraction seems to depend on the transverse momentum of the jets. In order to be able to compare these fractions with the measurements completed at the Tevatron, the CSE dijet fraction is plotted as a function of the $p_{T}$ of 

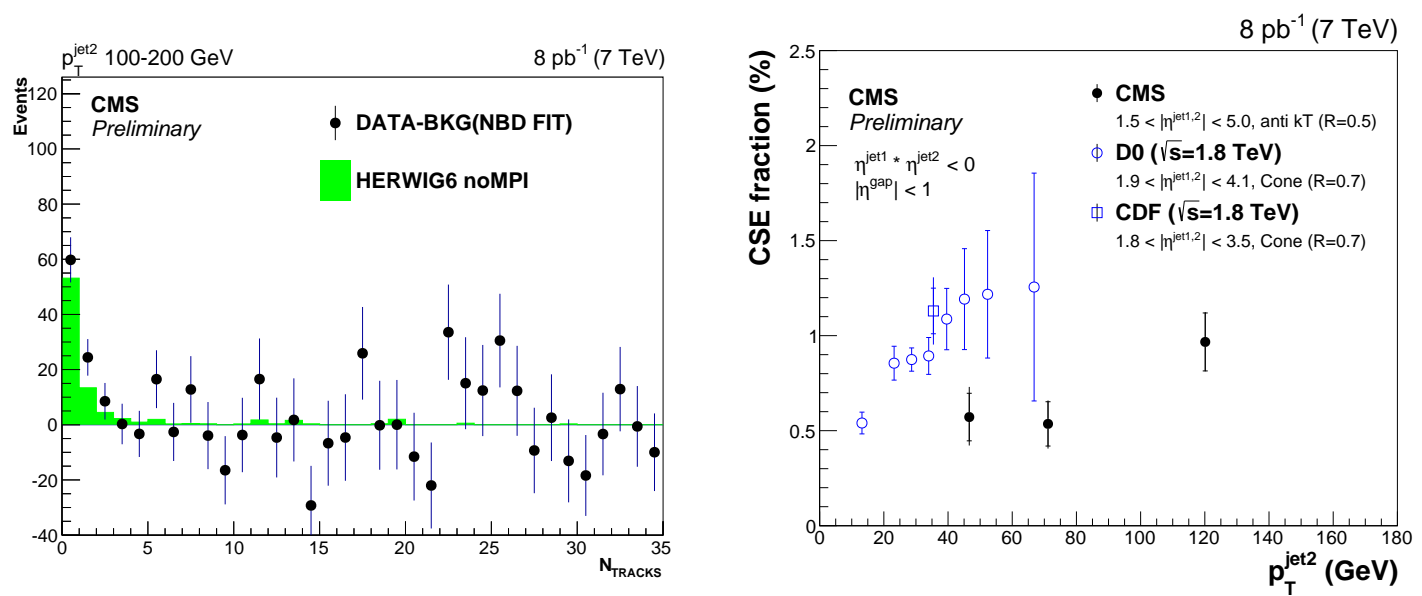

Figure 4: Left: Background-subtracted charged multiplicity distributions [2], compared to the HERWIG6 predictions for events without additional multi-parton interactions ("noMPI"). The background is estimated from the NBD fit to the data in the $3 \leq N_{\text {tracks }} \leq 35$ range, extrapolated to the lowest multiplicity bins. The normalization of the HERWIG6 MC is obtained from a fit to the data from Fig. 3. Right: The fraction $f_{\mathrm{CSE}}$ as a function of $p_{T}^{\text {jet2 }}$ as measured by CMS for $40<p_{T}^{\text {jet2 }}<60 \mathrm{GeV}, 60<p_{T}^{\text {jet2 }}<100 \mathrm{GeV}$, and $100<p_{T}^{\text {jet2 }}<$ $200 \mathrm{GeV}$ at $\sqrt{s}=7 \mathrm{TeV}$, and by the D0 and CDF experiments at $\sqrt{s}=1.8 \mathrm{TeV}$. The results are plotted at the mean value of $p_{T}^{\text {jet2 }}$ in each bin. The inner and outer error bars represent the statistical, and the statistical and systematic uncertainties added in quadrature, respectively.

the subleading jet on the right panel of Fig. 4. The CSE fraction slightly increases with transverse momentum, and decreases with increasing $\sqrt{s}$, indicating that there is probably more rescattering between spectator partons at higher collision energies, destroying the empty gap between the jets.

In summary, leading order DGLAP models do not reproduce dijet events and their properties where no particles are found between the jets. Evidence for CSE events was presented, and their fraction among dijet events measured, along with their dependence on transverse momentum and collision energy.

\section{References}

[1] CMS Collaboration, Azimuthal decorrelation of jets widely separated in rapidity in pp collisions at $\sqrt{s}=7 \mathrm{TeV}$, [arXiv:1601.06713 [hep-ex]].

[2] CMS Collaboration, Dijet production with a large rapidity gap between the jets, CMS Physics Analysis Summary FSQ-12-001.

[3] CMS Collaboration, The CMS experiment at the CERN LHC, JINST 3, S08004 (2008).

[4] B. Ducloué, L. Szymanowski and S. Wallon, Evidence for high-energy resummation effects in Mueller-Navelet jets at the LHC, Phys. Rev. Lett. 112 (2014) 082003 